\title{
Zika virus productively infects primary human placenta-specific macrophages
}

\author{
Kellie Ann Jurado, ${ }^{1}$ Michael K. Simoni, ${ }^{2}$ Zhonghua Tang, ${ }^{2}$ Ryuta Uraki, Jesse Hwang, ${ }^{1}$ \\ Sarah Householder, ${ }^{1}$ Mingjie Wu, ${ }^{1}$ Brett D. Lindenbach, ${ }^{3}$ Vikki M. Abrahams, ${ }^{2}$ Seth Guller, ${ }^{2}$ \\ and Erol Fikrig ${ }^{1,4}$ \\ ${ }^{1}$ Section of Infectious Diseases, Department of Internal Medicine, ${ }^{2}$ Department of Obstetrics, Gynecology and \\ Reproductive Sciences, and ${ }^{3}$ Department of Microbial Pathogenesis, Yale University School of Medicine, New Haven, \\ Connecticut, USA. ${ }^{4}$ Howard Hughes Medical Institute, Chevy Chase, Maryland, USA.
}

The strong association of Zika virus infection with congenital defects has led to questions of how a flavivirus is capable of crossing the placental barrier to reach the fetal brain. Here, we demonstrate permissive Zika virus infection of primary human placental macrophages, commonly referred to as Hofbauer cells, and placental villous fibroblasts. We also demonstrate Zika virus infection of Hofbauer cells within the context of the tissue ex vivo using term placental villous explants. In addition to amplifying infectious virus within a usually inaccessible area, the putative migratory activities of Hofbauer cells may aid in dissemination of Zika virus to the fetal brain. Understanding the susceptibility of placenta-specific cell types will aid future work around and understanding of Zika virus-associated pregnancy complications.

Conflict of interest: The authors have declared that no conflict of interest exists.

Submitted: May 6, 2016 Accepted: August 2, 2016 Published: August 18, 2016

Reference information: JCI Insight. 2016;1(13):e88461. doi:10.1172/jci.insight.88461.

\section{Introduction}

Analysis of a recent outbreak in Brazil linked Zika virus (ZIKV) infection of expecting mothers to microcephaly, a fetal developmental disorder in which the head and brain of the fetus/infant are smaller than usual (1). This is the first congenital defect attributed to infection by a member of the single-stranded RNA flavivirus genus. A causal relationship has not been firmly established; however, the entire ZIKV genome was recovered from the brain of a fetus from a pregnancy confirmed to be complicated by maternal ZIKV infection (2) and human embryonic cortical neural progenitor cells have been found to be particularly susceptible to ZIKV infection (3). Further, mouse models of vertical transmission have demonstrated ZIKV-induced fetal brain damage (4-6). In order for ZIKV to reach the fetal brain to cause organ-specific damage, it must cross the maternal-fetal barrier, which is composed of a syncytium from differentiation of underlying cytotrophoblasts. This multinuclear cell layer serves as a filter to prevent deleterious factors from reaching the fetus. Placental observations late in a ZIKV-infected pregnancy have shown abnormal calcifications by 32 weeks of gestation (2). Additionally, a mouse model of ZIKV-infected dams identified placental damage (4). If ZIKV is able to breach the maternal-fetal barrier and influence the placenta's integrity, there likely is a cell type within the placental villi that is permissive to ZIKV infection.

Primary human placental syncytiotrophoblasts, which separate the maternal blood from the fetal compartment, were recently described to resist ZIKV infection (7). Beyond the trophoblast syncytium and underlying mononuclear cytotrophoblast layer, the human placental villi also comprise mesenchymal cells, placental-specific macrophages/Hofbauer cells (HBCs), and fibroblasts (8). As macrophages have been identified as important target cells for dissemination of dengue virus (DENV), a related flavivirus (9), and immunohistochemistry of placental tissue from a confirmed ZIKV-complicated pregnancy suggested ZIKV infection of placental macrophages (10), we sought to determine ZIKV susceptibility of HBCs.

\section{Results}

Primary human HBCs and cytotrophoblast cells were isolated from the villous tissues of term placentas. HBC preparations were found to be $96 \%-97 \%$ pure, as indicated by flow cytometry for expression of macrophage marker CD163. To provide a thorough representation of circulating ZIKV isolates, 3 different strains of ZIKV were utilized for infection: the prototype Uganda/African strain MR766 from 1947 
A

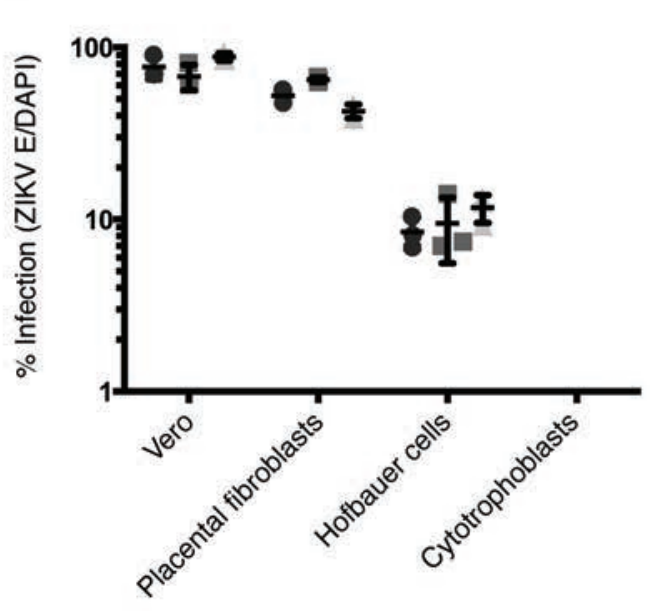

B
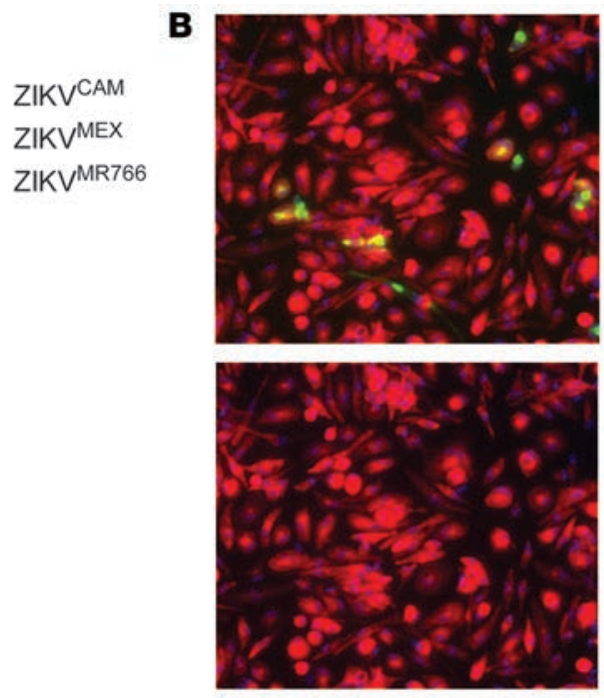

ZIKVCAM

ZIKVMEX

ZIKVMR766

D

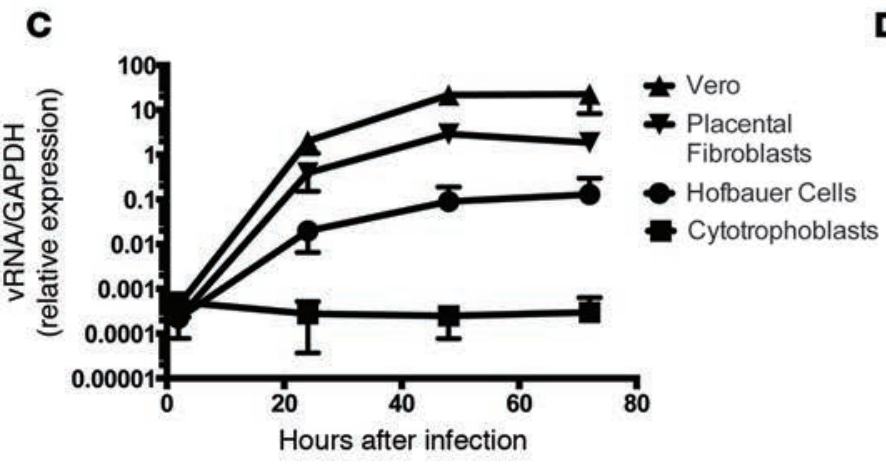

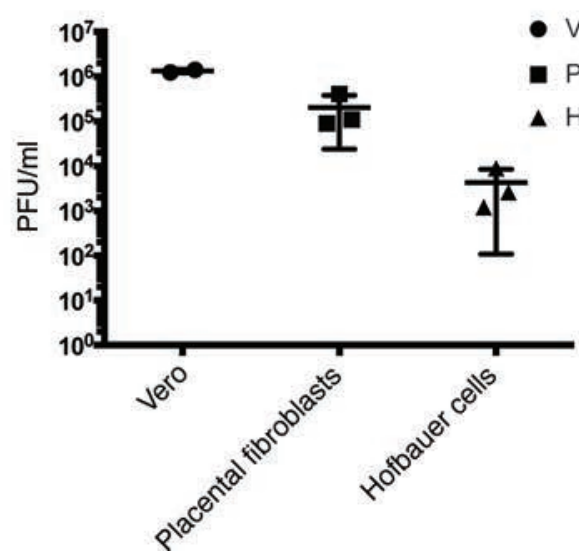

DAPI

CD 163

ZIKVE

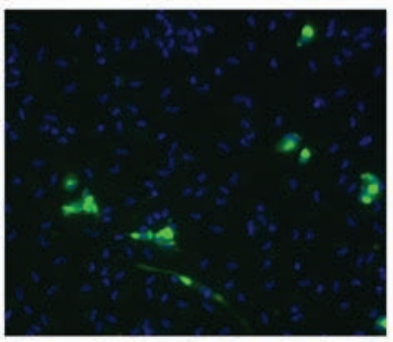

Vero

Placental Fibroblasts

Hofbauer Cells

Figure 1. Primary human placental-specific macrophages and fibroblasts are permissive to ZIKV infection. (A) The indicated primary placental cells and Vero cell line were infected with ZIKV CAM , ZIKV ${ }^{\text {MEX }}$, and ZIKVMR766 for 48 hours (MOI of 1), fixed, and stained with mouse monoclonal anti-ZIKV E (4C2). Data are shown as the percentage of positive ZIKV E cells relative to the total number of nuclei (as determined by DAPI) of an average of 3 biological replicates $( \pm$ SD) from 3 separate placenta preparations. (B) Representative immunofluorescence images of ZIKV-infected Hofbauer cells at $\times 20$ magnification. Infected cells were costained with anti-ZIKV E and anti-CD163 (host rabbit). (C) Viral growth kinetics of the indicated cells that were infected with an MOI of 1. Two hours after infection, cells were washed twice in PBS and fresh medium was applied. Quantitative PCR analysis was performed 2, 24, 48, and 72 hours after infection. Data are presented as the log relative expression of ZIKV RNA over GAPDH (average \pm SD, $n=3$ ). (D) Collected supernatants at 48 hours from C were assessed for the presence of infectious virus via plaque assays. Data are presented as log PFU/ml of supernatant (average \pm SD, $n=3$ ).

(ZIKV ${ }^{\mathrm{MR} 766}$ ), the FSS13025 Cambodian/Asian isolate from 2010 (ZIKV ${ }^{\mathrm{CAM}}$ ), and an Americas-derived virus isolated in 2016, MEX 2-81 (ZIKV ${ }^{\mathrm{MEX}}$ ).

The ZIKV-susceptible Cercopithecus aethiops kidney cell line, Vero, was used as a positive control in all in vitro experiments; these cultures demonstrated high infectivity (Figure 1A) and the characteristic viral replication kinetics (Figure 1C). Consistent with previous work in primary syncytiotrophoblast cells (7), we found that, at a MOI of 1 (Figure 1A), primary human trophoblast cells in isolated tissue cultures resisted a productive $\mathrm{ZIKV}$ infection regardless of the strain used. In contrast, colocalization of macrophage marker CD163 and the ZIKV envelope protein (E) indicated a permissive ZIKV infection of HBCs, with approximately $10 \%$ to $15 \%$ of cells positive for ZIKV antigen (Figure 1, A and B). Virus replication kinetics of ZIKV ${ }^{\text {CAM }}$ in HBCs confirmed productive infection of HBCs (Figure 1C), with a 3-log increase in cellular viral RNA by 48 hours. Further, the viruses shed from HBC infections were found to be infectious, as plaque assays of supernatants at 48 hours indicated an average titer of 3-log $\mathrm{PFU} / \mathrm{ml}$ within the culture media at 48 hours (Figure 1D). 


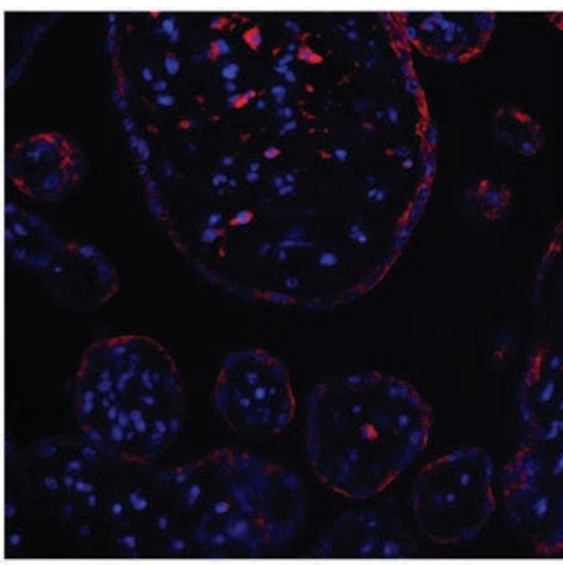

DAPI

dsRNA

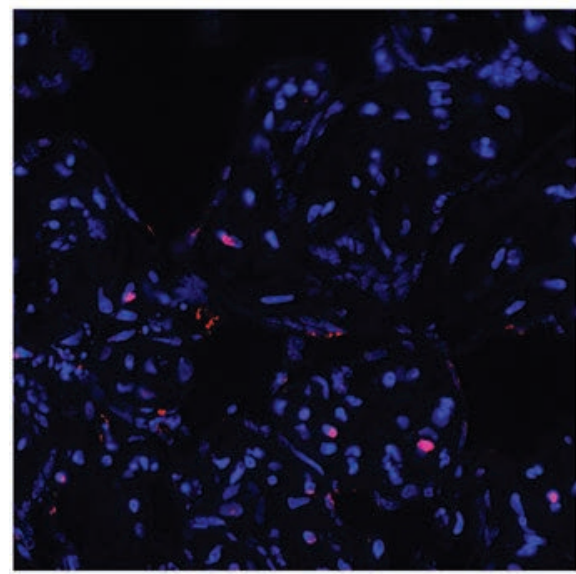

DAPI

NS1

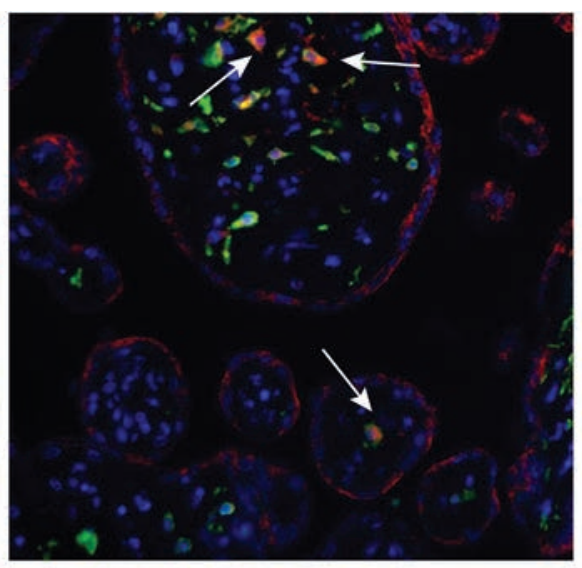

DAPI

dsRNA

CD 163

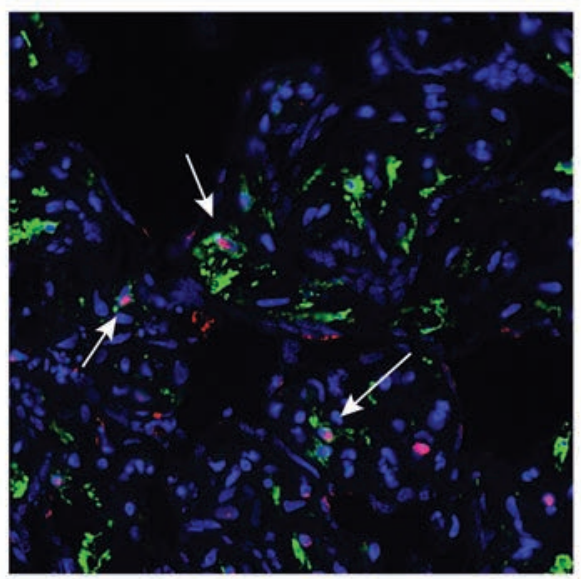

DAPI

NS1

CD 163

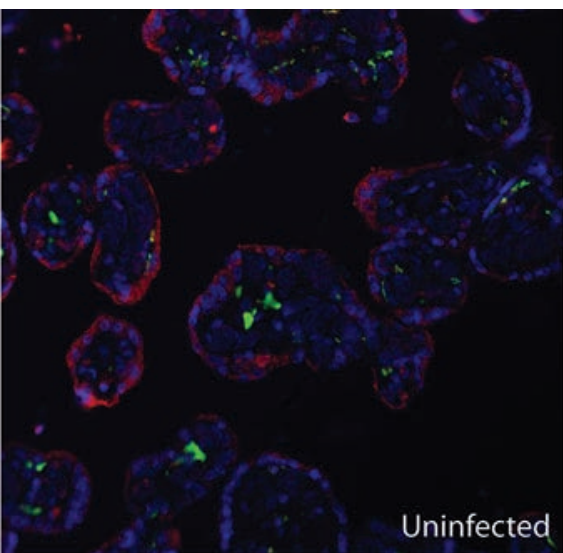

DAPI

dsRNA

CD 163

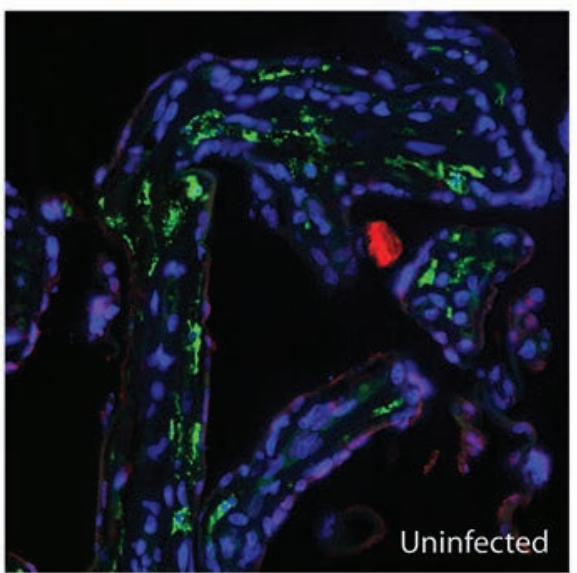

DAPI

NS1

CD 163

Figure 2. Primary human placental-specific macrophages are infected within the context of placental tissue. Villous tissue explants (60 mg) were infected with ZIKV within 2 hours of delivery, formalin fixed after 48 hours, and paraffin embedded. Sections were fluorescently labeled with mouse monoclonal $\mathrm{J} 2$ to detect viral dsRNA or NS1 to detect viral nonstructural protein and anti-CD163 macrophage cell marker. White arrows indicate infected HBCs.

Although examination by immunofluorescence unquestionably confirmed HBC susceptibility in vitro, there were few instances in which CD163-negative cells were infected; therefore, we next sought to determine whether primary human placental fibroblasts, which may persist within HBC preparations at low numbers, were additionally permissive to ZIKV infection. In contrast to the modest susceptibility of HBCs, placental fibroblasts in vitro were highly permissive to ZIKV challenge, with approximately 50\% of cells positive for ZIKV antigen at 48 hours (Figure 1A). Kinetics of ZIKVCAM replication confirmed productive infections of placental fibroblasts, with an approximately 5-log increase in cellular-associated viral RNA production. Plaque assays confirmed that infectious virus was shed by placental fibroblasts at 48 hours, with average titers of 5- to 6-log PFU/ml - levels of virus production similar to those in Vero cells.

To confirm the isolated primary cell culture findings within a more biologically relevant milieu, we next infected term placental villous explants with ZIKV ${ }^{\mathrm{CAM}}$ approximately 2 hours after delivery. Forty-eight hours after ZIKV infection, placental tissues were formalin fixed and paraffin embedded. Sections were cut and fluorescently costained with mouse monoclonal antibody, J2, to detect viral dsRNA as previously described (11) and macrophage cell marker CD163. Additionally, ZIKV protein was also probed with the ZIKV-infected placental explants utilizing an anti-NS1 mouse monoclonal antibody (12). Colocalization of anti-dsRNA and anti-NS1 with anti-CD163 confirmed HBC infection with an ex vivo infection in the context of placental tissue (white arrows, Figure 2). 


\section{Discussion}

Analysis by immunofluorescence, using monoclonal antibody, 4G2, that recognizes ZIKV E (13), suggested that cytotrophoblast cells do not replicate ZIKV ${ }^{\mathrm{CAM}}$, while RNA analysis of these infections revealed an interesting retention of input viral RNA within these cultured cells, even at 72 hours after infection (Figure 1C). Therefore, our data appear to suggest that virus replication is inhibited after entry within this cell population in vitro. Primary human cytotrophoblasts do undergo spontaneous cell-to-cell fusion when left in culture for longer than 48 hours (14); therefore, analyses of later time points with these cultured cells may reflect a mixed population of cytotrophoblasts and syncytiotrophoblasts.

Placental fibroblasts astonishingly exhibited similar efficiency of viral RNA replication as that seen in the interferon-deficient Vero cell line, which is commonly used to propagate flaviviruses (Figure 1C). Yet, the anti-dsRNA and anti-NS1 staining appeared to be more specific to CD163-positive cells within the milieu of the placental villi; therefore, infection of placental fibroblasts may be restricted within the context of tissue. The inconsistency in susceptibility of virus replication among isolated cultures and within the context of tissue may be due to a higher proliferative status of fibroblasts within isolated culture or perhaps resistance is due to the influence of cytokine excretion of surrounding cells. This finding serves as a cautionary note when using only isolated cell cultures/cell lines to interpret virus susceptibility.

As primary human trophoblast cells of term placentas appear to be resistant to a productive ZIKV infection, at least at early time points (up to 72 hours), breaching of the maternal-fetal barrier appears to be necessary for vertical transmission to transpire. Potential means by which virus can be transmitted across the maternal-fetal barrier are diverse (15) and may occur actively (exploiting a mechanism normally used to provide maternal aid to the fetus) or entail a passive event (surface tension resulting in breaks in the syncytium). Human cytomegalovirus (HCMV), a well-described causative agent of congenital birth defects, has been found to co-opt the neonate $\mathrm{Fc}_{\mathrm{c}}$ receptor-mediated IgG transport pathway ( $\mathrm{FcRn}$ ) to gain access into the fetal compartment by interacting with low neutralizing anti-HCMV maternal antibodies (16). DENV is hyperendemic in regions in which microcephaly has been associated with ZIKV infection (17), and DENV antibodies are known to have high serological cross-reactivity with ZIKV (18). It is intriguing to consider that preexisting circulating DENV antibodies within the mother could promote maternal antibody-mediated transcytosis of ZIKV across the resistant trophoblast layers to regions in which ZIKV-permissive cells (macrophages/fibroblasts) are actively surveying for signs of pathogens. These susceptible cells then serve as reservoirs for ZIKV within the placental compartment. We are currently developing models to test this hypothesis. If true, the congenital defects of ZIKV may be exacerbated within the Brazil outbreak by the presence of a population that has been previously exposed to DENV infections.

In summary, our data have shown that primary placental-specific fibroblasts and HBCs are permissive for ZIKV replication in isolated cultures in vitro while HBCs demonstrate susceptibility ex vivo in the context of placental tissues. Elucidation of the role that placental macrophages have during virus dissemination to the fetal brain will help our understanding of the pathogenesis of microcephaly and may lead to new strategies to prevent disease.

\section{Methods}

Placentas. Placentas $(n=3)$ were collected from scheduled elective cesarean sections without labor performed at Yale New Haven Hospital and brought to the laboratory within 30 minutes for preparation. Infection/inflammation was excluded on the basis of standard clinical criteria. Tissues were processed immediately for the isolation of placental cell cultures and explants. Each placenta was processed separately (i.e., tissues were not pooled).

Preparation of placental explants and isolation of placental cytotrophoblasts, HBCs, and fibroblasts. Isolation of HBCs and cytotrophoblasts from the same placenta was carried out as previous described (19) with slight modifications. $60 \mathrm{mg}$ of minced placental tissue was rinsed in PBS and placed in a 24-well plate filtered with 0.4- and 3.0- $\mu \mathrm{m}$ permeable cell culture inserts (BD Falcon). For cell isolations, villous tissue was initially digested with trypsin/deoxyribonuclease I digestion, and then the digestate was centrifuged on a discontinuous Percoll gradient (50\%/45\%/35\%/30\%). Cells migrating to the $35 \% / 45 \%$ Percoll interface were recovered and immunopurified by negative selection by simultaneous treatment with magnetic beads conjugated to mouse anti-human CD9 antibody and mouse anti-human CD45 antibody. HBCs were isolated from trypsin-treated tissue fragments that were further digested with collagenase A and loaded onto a discontinuous Percoll gradient (40\%/35\%/30\%/20\%). Cells from the $20 \% / 30 \%$ to $30 \% / 35 \%$ interfaces 
were recovered. Cells were plated, and after 30 minutes, floating and weakly adherent cells were removed and discarded. Purity of individual HBC preparations was determined by flow cytometry.

Cultures of placental fibroblasts were obtained using cells attached to magnetic beads containing CD9/ CD45 antibody from cytotrophoblast preparations (19). The bead-cell mixtures were washed and cultured using a 1:1 mixture of DMEM/F12 with $10 \%$ FBS and $1 \%$ antibiotic-antimycotic (i.e., FBS medium). Fresh FBS medium was added every 2 to 3 days until confluency was reached. After trypsinization of first-passage cells, magnetic beads with attached cells, comprising approximately $10 \%$ of the cell population, were removed with a magnet and discarded.

Cell lines and virus strains. Vero cells (ATCC) were maintained in DMEM containing 10\% FBS and antibiotics at $37^{\circ} \mathrm{C}$ with $5 \% \mathrm{CO}_{2}$ and have been routinely confirmed to be mycoplasma free. Aedes albopictus midgut C6/36 cells were grown in DMEM supplemented with 10\% FBS, 1\% tryptose phosphate, and antibiotics at $30^{\circ} \mathrm{C}$ with $5 \% \mathrm{CO}_{2}$ air atmosphere. $\mathrm{ZIKV}^{\mathrm{CAM}}$ and $\mathrm{ZIKV}{ }^{\mathrm{MEX}}$ were obtained from the University of Texas Medical Branch at Galveston's World Reference Center for Emerging Viruses and Arboviruses and propagated in $\mathrm{C} 6 / 36$ insect cells. ZIKV ${ }^{\mathrm{MR} 766}$ was acquired from BEI Resources and propagated in Vero cells. Viral titers were determined utilizing plaque assays as described below.

Immunofluorescence. Cells were fixed in 4\% paraformaldehyde, washed with PBS, disrupted with $0.1 \%$ Triton X-100 (American Bioanalytical) in PBS, and blocked in 1\% BSA and 0.2\% Tween20 in PBS. ZIKV $\mathrm{E}$ was probed with monoclonal anti-flavivirus group antigen antibody 4G2 (Millipore) at a 1:1,000 dilution. CD163 antibody (Abcam, EPR14643-36) was used at 1:500 to identify HBCs. Fluorescence was visualized with a Zeiss Axiovert $200 \mathrm{M}$ fluorescence microscope, and images were analyzed with ImageJ (NIH).

$R N A$. Total RNA was extracted from cultured cells using the Qiagen RNeasy Mini Kit and reverse-transcribed using the iScript cDNA Synthesis Kit (Bio-rad) according to manufacturer's protocol. Gene expression was queried using IQ SYBR Green Supermix (Bio-Rad). Gene expression was calculated using the 2- $\Delta \Delta \mathrm{CT}$ method normalized to GAPDH.

Plaque assay. Vero cell monolayers were incubated with cell-free supernatants of cultures from growth curve experiments, which were collected at 48 hours, or with cell-free supernatant from viral propagations in 10 -fold serial dilutions for 1 hour at $37^{\circ} \mathrm{C}$ and overlaid with a mixture of $2 \%$ agarose and $2 \times$ media. 3 to 4 days post infection, cells were fixed by $10 \%$ formalin, stained with $0.005 \%$ amido black and PFU were counted.

Immunohistochemistry. $60 \mathrm{mg}$ of minced placental tissue was infected within 2 hours by direct addition to the insert of ZIKV ${ }^{\text {CAM }}$. Tissue was rinsed in PBS prior to fixation by $10 \%$ neutral-buffered formalin. Fixed tissue was submitted to the Yale Pathology Tissue Services core at Yale School of Medicine for further processing with J2 staining as previously described (11). NS1 staining of FFPE tissue was completed by using mouse monoclonal antibody 17A12-1G5, which was originally raised against DENV-2 NS1 (12), and produced though intraperitoneal injection of the hybridoma and collection of ascetic fluid. NS1 antigen retrieval was completed by boiling antigen in citrate buffer ( $\mathrm{pH}$ 6) for 20 minutes followed by overnight incubation at a 1:1,000 dilution. Anti-rabbit CD163 (Abcam) was used at a 1:200 dilution.

Study approval. Approval for this study was granted by the Yale University School of Medicine Human Investigation Committee. Informed consent was not necessary as all samples were discardable tissue and deidentified.

\section{Author contributions}

KAJ and MKS conceived and designed experiments. ZT isolated primary human placental tissues and cells. KAJ, MKS, RU, SH, and MW organized and conducted experiments. EF, JH, SG, BDL, and VMA contributed to discussions and provided feedback throughout the project. KAJ and MKS wrote the paper, and all the authors reviewed and/or modified the manuscript.

\section{Acknowledgments}

We thank Marie Flamand (Institut Pasteur) for the contribution of the NS1 mouse monoclonal antibody. This work was supported by NIH grant 5T32 AI007019-40 (to KAJ) and the Albert McKern Scholar Award (to SG). EF is an investigator with the Howard Hughes Medical Institute.

Address correspondence to: Erol Fikrig, Section of Infectious Diseases, Department of Internal Medicine. Yale University School of Medicine, The Anlyan Center for Medical Research and Education, 300 Cedar Street, New Haven, Connecticut 06520, USA. Phone: 203.785.4140; E-mail: erol.fikrig@yale.edu. 
1. Rasmussen SA, Jamieson DJ, Honein MA, Petersen LR. Zika virus and birth defects - reviewing the evidence for causality. $N$ Engl J Med. 2016;374(20):1981-1987.

2. Mlakar J, et al. Zika virus associated with microcephaly. N Engl J Med. 2016;374(10):951-958.

3. Tang H, et al. Zika virus infects human cortical neural progenitors and attenuates their growth. Cell Stem Cell. 2016;18(5):587-590.

4. Miner JJ, et al. Zika virus infection during pregnancy in mice causes placental damage and fetal demise. Cell. 2016;165(5):1081-1091.

5. Li C, et al. Zika virus disrupts neural progenitor development and leads to microcephaly in mice. Cell Stem Cell. 2016;19(1):120-126.

6. Cugola FR, et al. The Brazilian Zika virus strain causes birth defects in experimental models. Nature. 2016;534(7606):267-271.

7. Bayer A, et al. Type III interferons produced by human placental trophoblasts confer protection against Zika virus infection. Cell Host Microbe. 2016;19(5):705-712.

8. Wang Y, Zhao S, eds. Cell types of the placenta. In: Vascular Biology of the Placenta. San Rafael, California, USA: Morgan \& Claypool Life Sciences; 2010:Chapter 4.

9. Halstead SB. Antibody, macrophages, dengue virus infection, shock, and hemorrhage: a pathogenetic cascade. Rev Infect Dis. 1989;11(supp1 4):S830-S839.

10. Noronha Ld, Zanluca C, Azevedo ML, Luz KG, Santos CN. Zika virus damages the human placental barrier and presents marked fetal neurotropism. Mem Inst Oswaldo Cruz. 2016;111(5):287-293.

11. Richardson SJ, et al. Use of antisera directed against dsRNA to detect viral infections in formalin-fixed paraffin-embedded tissue. J Clin Virol. 2010;49(3):180-185.

12. Schul W, Liu W, Xu HY, Flamand M, Vasudevan SG. A dengue fever viremia model in mice shows reduction in viral replication and suppression of the inflammatory response after treatment with antiviral drugs. J Infect Dis. 2007;195(5):665-674.

13. Hamel R, et al. Biology of Zika virus infection in human skin cells. J Virol. 2015;89(17):8880-8896.

14. Dodeur M, Malassine A, Bellet D, Mensier A, Evain-Brion D. Characterization and differentiation of human first trimester placenta trophoblastic cells in culture. Reprod Nutr Dev. 1990;30(2):183-192.

15. Delorme-Axford E, Sadovsky Y, Coyne CB. The placenta as a barrier to viral infections. Annu Rev Virol. 2014;1(1):133-146.

16. Maidji E, McDonagh S, Genbacev O, Tabata T, Pereira L. Maternal antibodies enhance or prevent cytomegalovirus infection in the placenta by neonatal Fc receptor-mediated transcytosis. Am J Pathol. 2006;168(4):1210-1226.

17. Schuler-Faccini L, et al. Possible association between Zika virus infection and microcephaly - Brazil, 2015. MMWR Morb Mortal Wkly Rep. 2016;65(3):59-62

18. Brasil P, et al. Zika virus outbreak in Rio de Janeiro, Brazil: clinical characterization, epidemiological and virological aspects. PLoS Negl Trop Dis. 2016;10(4):e0004636.

19. Tang Z, Tadesse S, Norwitz E, Mor G, Abrahams VM, Guller S. Isolation of Hofbauer cells from human term placentas with high yield and purity. Am J Reprod Immunol. 2011;66(4):336-348. 